\title{
Diffusion-weighted MR imaging of normal and abnormal scrotum: preliminary results
}

\author{
Athina C Tsilii ${ }^{1}$, Maria I Argyropoulou ${ }^{1}$, Dimitrios Giannakis ${ }^{2}$, Stavros Tsampalas ${ }^{2}$, Nikolaos Sofikitis ${ }^{2}$ \\ and Konstantinos Tsampoulas ${ }^{1}$
}

Magnetic resonance (MR) imaging of the scrotum represents an important supplemental diagnostic tool in the evaluation of scrotal diseases. Diffusion-weighted (DW) MR imaging is a developing technique, proved to improve tissue characterization. We evaluated the feasibility and diagnostic performance of DW MR imaging in the detection and characterization of scrotal lesions. We retrospectively evaluated 31 scrotal lesions ( 23 intratesticular and 8 extratesticular) in 26 men. All MR examinations were performed on a 1.5-T unit, using a pelvic-phased array coil. DW sequences were obtained using a single shot, multislice spin echo planar diffusion pulse sequence and a $b$ factor of 0 and $900 \mathrm{~s} \mathrm{~mm}^{-2}$. The DW MR characteristics and the apparent diffusion coefficient (ADC) values of normal scrotal contents and scrotal diseases were evaluated. Comparison between the ADC values of normal scrotum, benign lesions and scrotal malignancies was performed. The accuracy of conventional sequences, DW images alone and DW imaging combined with conventional images in differentiating benign from malignant scrotal lesions was calculated. The ADC values of testicular malignancies were different from those of normal testis and benign intratesticular lesions, and the ADC values of benign extratesticular lesions from those of normal epididymis $(\boldsymbol{P}<0.05)$. The overall accuracy of conventional imaging, DW imaging alone and DW MR combined with conventional sequences in the characterization of intratesticular lesions was $91 \%, 87 \%$ and $100 \%$, respectively. Our findings suggest that DW MR imaging and ADC values may provide valuable information in the diagnosis and characterization of scrotal diseases. Asian Journal of Andrology (2012) 14, 649-654; doi:10.1038/aja.2011.172; published online 27 February 2012

Keywords: diagnosis; epididymis; male genital neoplasms; scrotum; testicular neoplasms; testis

\section{INTRODUCTION}

Although sonography remains the primary imaging modality in the diagnosis of scrotal pathology, ${ }^{1,2}$ magnetic resonance (MR) imaging of the scrotum may provide valuable information in the detection and characterization of various scrotal disorders. ${ }^{3-9}$ MR evaluation of the scrotum performs well in localizing scrotal masses, therefore differentiating intratesticular and extratesticular scrotal disorders. A preoperative characterization of the histological nature of various scrotal masses is also possible in many cases. ${ }^{3-9}$

Diffusion-weighted (DW) MR imaging watches the random microscopic motion of water molecules (Brownian motion). ${ }^{10,11}$ Recently, the technique proved useful in the detection of malignant neoplasms and the histological characterization of focal lesions in the abdomen. ${ }^{10,11}$ Lesion detection and characterization is largely depended by the extent of tissue cellularity, and increased cellularity is associated with restricted diffusion and reduced apparent diffusion coefficient (ADC) values. The ADC values of malignancies are reported lower than those of benign lesions or normal tissues. ${ }^{10-23}$ Published series reported the usefulness of DW MR imaging in the early detection of ischemia in the testis of Wistar rats and in the detection of nonpalpable testes in children. ${ }^{24,25}$ To the author's knowledge, there are no reports in the English-language literature, regarding the normal ADC values of the scrotal contents, that is the testis and the epididymis and the ADC values of scrotal pathology. The purpose of this study was to determine the DW MR imaging characteristics and ADC values of normal scrotal contents and various scrotal disorders and to assess the feasibility and diagnostic performance of DW MR imaging in the characterization of scrotal lesions.

\section{MATERIALS AND METHODS}

Study population

This was a retrospective study including 39 patients (age range, 20-81 years; mean age, 44 years). Thirteen men were presented with vague scrotal pain, and normal sonographic examination of the scrotum, both on admission and follow-up. From the remaining, 18 patients were referred for painless scrotal enlargement and/or clinically and sonographically detected scrotal mass, in one case following a recent trauma, three patients for painless enlargement of the scrotum, in one case after a recent testicular biopsy, and five patients with signs of epididymo-orchitis. Due to the retrospective nature of the study, the institutional review board did not require approval or patients' informed consent.

\section{MR imaging protocol}

All patients were scanned in the supine position on a 1.5-T MR system (Intera; Philips Medical Systems, Cleveland, OH, USA), using a pelvic

${ }^{1}$ Department of Clinical Radiology, University Hospital of loannina, Ioannina 45500, Greece and 2Department of Urology, University Hospital of loannina, Ioannina 45500, Greece Correspondence: Dr. AC Tsili (a_tsili@yahoo.gr)

Received: 5 September 2011; Revised: 21 October 2011; Accepted: 9 November 2011; Published online: 27 February 2012 
phased-array coil. A towel was placed under the scrotum to keep a comparable distance of both testicles from the coil and the penis was draped on the anterior abdominal wall. The MR protocol used is illustrated on Table 1. Axial spin echo T1-weighted sequences, and axial, sagittal and coronal fast spin echo $\mathrm{T} 2$-weighted images were obtained. Axial fat-suppressed T1-weighted sequences were repeated when a lesion with high T1 signal intensity was noted. DW imaging was performed along the axial plane, using a single shot, multislice spin echo planar diffusion pulse sequence and $b$ values of 0 and $900 \mathrm{~s} \mathrm{~mm}^{-2}$. The orientation and location of these images were identical to the conventional transverse sequences. The time required to acquire the DW sequences was $29 \mathrm{~s}$. In patients with scrotal pathology, gadolinium-DTPA (Omniscan $\left(0.2 \mathrm{mmol} \mathrm{kg}^{-1}\right)$; Amersham Health, Oslo, Norway) was administered intravenously. Dynamic contrast-enhanced MR imaging in the coronal plane and transverse spin echo contrast-enhanced T1-weighted images were subsequently obtained.

\section{MR imaging data interpretation}

Two radiologists read the MR imaging data, without the knowledge of clinical information or histopathological diagnosis. Any discrepancy was resolved in consensus. The following items were evaluated: (i) the lesion signal intensity on conventional plain and contrast-enhanced MR images; and (ii) the signal intensity of intratesticular and/or paratesticular mass lesions on DW images, compared to that of the normal testicular parenchyma and the epididymis, respectively. Subsequently, DW images were read in conjunction with the transverse T2-weighted images, and ADC maps were created on a workstation (MxView; Philips Medical Systems), after zooming the image by a factor of 1.5. For the quantitative analysis, a single radiologist defined a circular region of interest to be as large as possible within the scrotal lesion, excluding areas of hemorrhage or necrosis, with the aid of the corresponding transverse T1- and T2-weighted images. Three measurements were obtained and averaged. A similar region of interest placed in the middle of the testis, encompassing the majority of testicular parenchyma was also drawn to calculate the ADC values of the normal testis. Calculations of the normal values of the epididymis were obtained when the epididymal parts were readily identifiable in the corresponding transverse T2-weighted images.

At first, MR imaging data were interpreted on the conventional sequences and a possible diagnosis of benignity or malignancy was written. Subsequently, DW images alone and DW sequences in conjunction with the plain and contrast-enhanced MR sequences were evaluated. The accuracy of conventional MR imaging data alone,
DW imaging alone and conventional sequences combined with DW sequences in the characterization of scrotal lesions was calculated. The standard of reference included clinical and imaging follow-up, surgical and histopathological findings. The time interval between MR imaging and surgery (radical orchiectomy was performed in 15 patients and tumor excision in 1 case) was less than 2 weeks in all cases.

\section{Statistical analysis}

Statistical analysis was performed with SPSS Base 16 for windows (SPSS Inc., Chicago, IL, USA). The normality of distribution of the parameters was assessed using the Kolmogorov-Smirnov test. The ADC values of testicular carcinoma were compared with those of normal testicular parenchyma and benign intratesticular lesions using ANOVA followed by the least significance difference test. Independent samples $t$-test was used to study differences between the ADC values of the various paratesticular lesions with those of the normal epididymis. A $P$ value of less than 0.05 was considered statistically significant. The sensitivity, specificity and accuracy of conventional MR images alone, DW images and DW imaging combined with the conventional sequences were calculated.

\section{RESULTS}

The final diagnoses were 31 scrotal disorders in 26 men, including 23 intratesticular lesions, 14 (61\%) of which were malignant and $9(39 \%)$ benign and 8 extratesticular diseases, all (100\%) proved benign. These diagnoses are shown in Table 2.

MR evaluation included 78 testicular units, 55 (70\%) of which characterized as normal and $23(30 \%)$ as abnormal. Normal testicular parenchyma appeared hyperintense on both T2-weighted and DW images, and slightly hypointense on the ADC maps (Figure 1). The mean \pm s.d. of ADC values $\left(\times 10^{-3} \mathrm{~mm}^{2} \mathrm{~s}^{-1}\right)$ of the normal testis were $1.11 \pm 0.18$.

Conventional MR images enabled the correct characterization of all (100\%) intratesticular malignancies and seven out of nine (78\%) cases of benign testicular lesions. The technique had a sensitivity of $100 \%$, a specificity of $78 \%$, a positive predictive value of $87.5 \%$, a negative predictive value of $100 \%$ and an overall accuracy of $91 \%$ in differentiating malignant from benign testicular lesions. Intratesticular malignancies were diagnosed using the MR criteria known from previously published reports. ${ }^{7,26,27}$ Testicular carcinomas were detected as either of low $(n=9)$ or heterogeneous $(n=5)$ signal intensity on T2-weighted images, with areas of hemorrhage $(n=5)$ or necrosis $(n=5)$, all heterogeneously enhancing after contrast material administration (Figures 2

Table 1 MR protocol (1.5-T unit) used for the evaluation of scrotal diseases

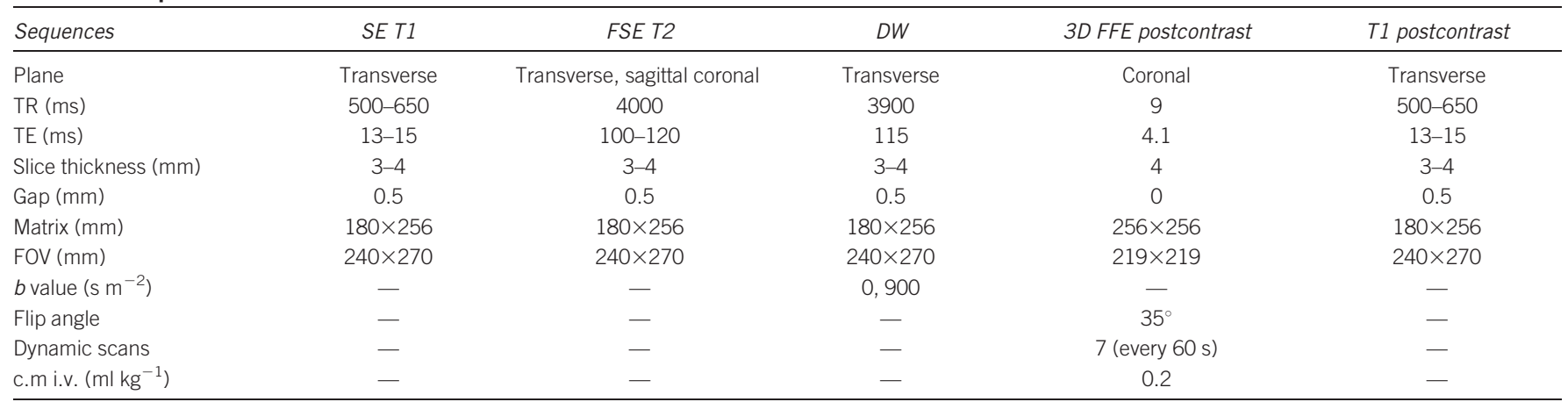

Abbreviations: c.m. i.v., contrast medium intravenously; DW, diffusion-weighted; FOV, field of view; FSE, fast spin echo; MR, magnetic resonance; SE, spin echo; 3D FFE: 3-dimensional fast field echo; TE: echo time; TR: repetition time. —: Not measured. 
Table 2 Diagnoses of intratesticular and extratesticular diseases

\begin{tabular}{lc}
\hline Diagnosis & Number \\
\hline Intratesticular lesions & 23 \\
Malignant & 14 \\
Seminomas & 8 \\
Nonseminomatous germ cell tumors & 6 \\
Benign & 9 \\
Benign Sertoli cell tumor & 1 \\
Acute epididymo-orchitis & 3 \\
Post-biopsy changes & 1 \\
Post-traumatic hematoma & 1 \\
Tubular ectasia rete testis & 3 \\
Extratesticular lesions & 8 \\
Benign & 8 \\
Acute epididymitis/epididymo-orchitis & 5 \\
spermatocele & 2 \\
Adenomatoid tumor of the tunica albuginea & 1 \\
\hline
\end{tabular}

and 3). There was also invasion of the testicular tunicae by the neoplasm in two cases, invasion of the epididymis in one case and extension of the tumor to the spermatic cord in three patients (Figure 2).

The MR features of tubular ectasia of rete testis were typical in this series, including the presence of multicystic masses, involving the area of the mediastinum testis, with signal intensity identical to that of water that is hypointense and hyperintense on T1- and T2-weighted images, respectively, not enhancing after contrast material administration. ${ }^{28,29}$ The diagnosis of intratesticular hematoma was straightforward in our patient, based on the presence of a hyperintense mass lesion on both T1- and T2-weighted sequences and lack of contrast enhancement. Five cases of epididymo-orchitis/epididymitis were also correctly characterized by conventional MR images. Enlargement and contrast enhancement of the epididymis and/or the testis, combined with signal hypointensity on T2-weighted images (Figure 4) were findings proved to correspond to acute inflammation on clinical and sonographic follow-up in this study.

We had two false positives in this material, detected as inhomogeneously contrast-enhancing lesions on conventional MR sequences (Figure 5). One of these cases proved histologically to represent a benign Sertoli cell tumor (Figure 5), and in the other case postbiopsy changes were the cause of intratesticular lesion, as proved by the clinical history and subsequent sonographic follow-up.

DW images alone had a sensitivity of $85.7 \%$, a specificity of $88.8 \%$, a positive predictive value of $92.3 \%$, a negative predictive value of $80 \%$ and an accuracy of $87 \%$ in the characterization of intratesticular

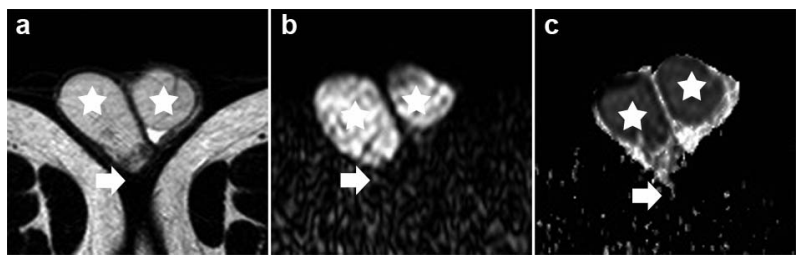

Figure 1 Normal testis and epididymis in a 21-year-old man. (a) Transverse T2weighted image shows normal hyperintensity of both testicles (asterisk). The tail of the right epididymis is hypointense (arrow), when compared to the normal testicular parenchyma. (b) Transverse diffusion-weighted echo planar image ( $b=900$ $\mathrm{s} \mathrm{mm}^{-2}$ ) depicts normal testicular parenchyma with high signal intensity (asterisk) and right epididymal tail with low signal intensity (arrow). (c) The ADC values of testicular parenchyma were $0.87 \times 10^{-3} \mathrm{~mm}^{2} \mathrm{~s}^{-1}$ (right testis, asterisk) and $0.82 \times 10^{-3} \mathrm{~mm}^{2} \mathrm{~s}^{-1}$ (left testis, asterisk) and the ADC value of the right epididymis was $1.54 \times 10^{-3} \mathrm{~mm}^{2} \mathrm{~s}^{-1}$ (arrow). ADC, apparent diffusion coefficient.

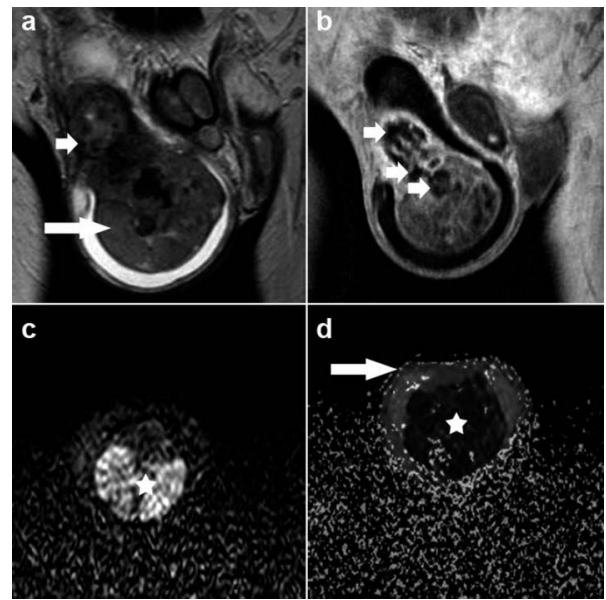

Figure 2 Classic right testicular seminoma with extension to the spermatic cord in a 58-year-old man. (a) Coronal T2-weighted image depicts a large inhomogeneous tumor of the right testis, mainly hypointense, extending to the lower part of the spermatic cord (arrow). A moderate right hydrocele (long arrow) is also seen. (b) Coronal dynamic contrast-enhanced image (early phase) shows strong and heterogeneous enhancement. Areas that do not enhance (small arrows) correspond to areas of necrosis, as proved on pathology. (c) Transverse DW echo planar image ( $b=900 \mathrm{~s} \mathrm{~mm}^{-2}$ ) demonstrates tumor hypeintensity (asterisk). (d) The ADC values of testicular carcinoma were $0.7 \times 10^{-3} \mathrm{~mm}^{2} \mathrm{~s}^{-1}$ (asterisk), lower than that of the normal contralateral testis $\left(1.29 \times 10^{-3} \mathrm{~mm}^{2} \mathrm{~s}^{-1}\right)$ and the ipsilateral hydrocele $\left(2.74 \times 10^{-3} \mathrm{~mm}^{2} \mathrm{~s}^{-1}\right.$, long arrow). ADC, apparent diffusion coefficient; DW, diffusion-weighted.

masses. On DW imaging, all but two testicular malignancies were clearly depicted as areas of restricted diffusion that is hyperintense, compared to the normal testicular parenchyma (Figures $2 \mathrm{c}$ and $3 \mathrm{c}$ ) and hypointense (Figures $\mathbf{2} \mathbf{d}$ and $\mathbf{3 d}$ ) on the DW images and ADC maps, respectively. All but one benign testicular lesions were detected
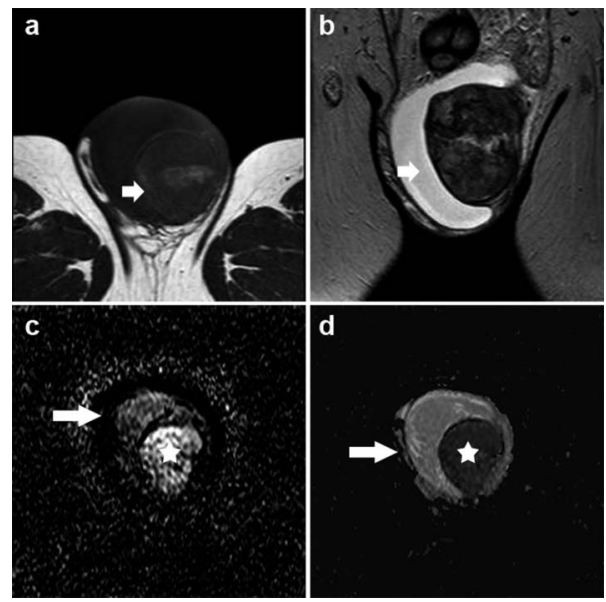

Figure 3 Embryonal carcinoma of the right testis, with invasion of the testicular tunicae and the ipsilateral epididymis (not shown in images) in a 41-year-old man. (a) Transverse T1- and (b) coronal T2-weighted images depict a heterogeneous mass, replacing the right testis. Hyperintensity (arrow) on T1-weighted images within the tumor corresponded to hemorrhagical foci on histology. (c) Transverse DW echo planar image $\left(b=900 \mathrm{~s} \mathrm{~mm}^{-2}\right)$ shows restricted tumor diffusion. (d) The ADC values of testicular malignancy (asterisk) were $0.85 \times 10^{-3} \mathrm{~mm}^{2} \mathrm{~s}^{-1}$, lower than that of the normal left testicular parenchyma $\left(1.32 \times 10^{-3} \mathrm{~mm}^{2} \mathrm{~s}^{-1}\right)$. A large right hydrocele, with an ADC value of $2.7 \times 10^{-3} \mathrm{~mm}^{2} \mathrm{~s}^{-1}$ is also revealed (long arrow). ADC, apparent diffusion coefficient; DW, diffusion-weighted. 


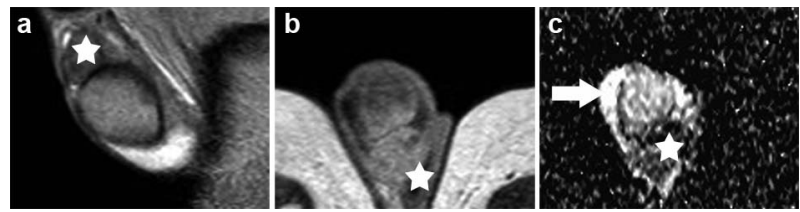

Figure 4 Right epididymo-orchitis in a 23-year-old man, with typical findings on MR imaging. (a) Sagittal T2-weighted and (b) transverse contrast-enhanced T1weighted images demonstrate enlargement and hypointensity of the right epididymis, with strong enhancement (asterisk). The right testis was also enlarged, enhancing more than the normal contralateral testis (not shown on images). (c) The ADC values of the right epididymis were $1.53 \times 10^{-3} \mathrm{~mm}^{2} \mathrm{~s}^{-1}$ (left epididymis: $1.37 \times 10^{-3} \mathrm{~mm}^{2} \mathrm{~s}^{-1}$ ) and that of the right testis $1.08 \times 10^{-3} \mathrm{~mm}^{2} \mathrm{~s}^{-1}$, (left testis: $1.37 \times 10^{-3} \mathrm{~mm}^{2} \mathrm{~s}^{-1}$ ). A small amount of hydrocele (long arrow), with an ADC value of $2.52 \times 10^{-3} \mathrm{~mm}^{2} \mathrm{~s}^{-1}$ is also seen in the right hemiscrotum. ADC, apparent diffusion coefficient; MR, magnetic resonance.

without causing significant restricted diffusion (Figure 4), therefore of low signal intensity on DW sequences. The mean \pm s.d. of ADC values $\left(\times 10^{-3} \mathrm{~mm}^{2} \mathrm{~s}^{-1}\right)$ of intratesticular malignancies and benign intratesticular lesions were $0.85 \pm 0.62$ and $1.56 \pm 0.85$, respectively. ANOVA analysis between normal testis, benign and malignant intratesticular lesions showed that the mean ADC values were different $(F=7.83, P<0.001)$ (Table 3). The least significance difference test showed a difference between the ADC values of normal testicular parenchyma and testicular malignancies $(P=0.032)$, the ADC values of benign and malignant intratesticular lesions $(P=0.000)$, and between the measurements of normal testis and benign intratesticular lesions $(P=0.005)$.

Based on the DW data alone, this study had one false positive in a patient with intratesticular hematoma, detected with restricted diffusion on DW images and very low ADC values. Although DW findings were suggestive of malignancy, conventional MR sequences enabled the correct characterization in this patient. There were also two false negatives, including multicystic intratesticular malignancies, without obviously detectable areas of restricted diffusion on imaging. However, conventional MR features were typical for malignancy in these patients, as subsequently proved on histopathology.

The interpretation of DW data combined with conventional images enabled the correct characterization of both false positives, based on the interpretation of the conventional data alone in this study. Both benign Sertoli cell tumor (Figure 5c) and postbiopsy changes were hypointense on DW imaging, therefore not confirming the diagnosis of malignancy. The combined evaluation of both conventional and
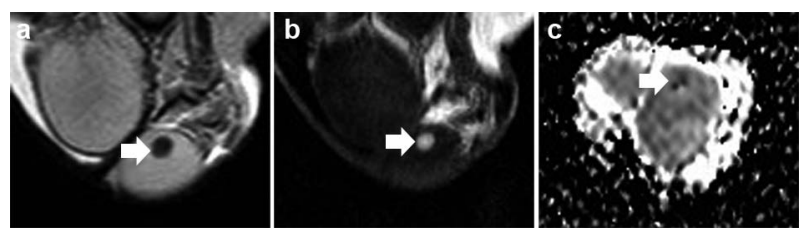

Figure 5 Benign Sertoli cell tumor of the left testis in a 22-year-old man. Coronal (a) T2-weighted and (b) dynamic contrast-enhanced images (early phase) show a small upper pole intratesticular mass (arrow). The lesion is ill-defined, hypointense on T2-weighted images, with strong enhancement. Conventional MR images were suggestive for malignancy. (c) Transverse DW image ( $b=700 \mathrm{~s}$ $\mathrm{mm}^{-2}$ ) depicts tumor hypointensity (arrow); therefore, the diagnosis of benignity could not be excluded. ADC, apparent diffusion coefficient; DW, diffusionweighted; MR, magnetic resonance.
Table 3 Analysis of variance between the ADC values of normal testicular parenchyma, benign and malignant intratesticular lesions

\begin{tabular}{lccccc}
\hline & $\begin{array}{c}\text { Normal } \\
\text { testis } \\
(\mathrm{n}=55)\end{array}$ & $\begin{array}{c}\text { Benign } \\
(\mathrm{n}=8)\end{array}$ & $\begin{array}{c}\text { Malignant } \\
(\mathrm{n}=14)\end{array}$ & $\mathrm{F}$ & $\mathrm{P}$ \\
\hline $\begin{array}{c}\text { Mean ADC } \pm \mathrm{s} . \mathrm{d} . \\
\left(\times 10^{-3} \mathrm{~mm}^{2} \mathrm{~s}^{-1}\right)\end{array}$ & $1.11 \pm 0.18$ & $1.56 \pm 0.85$ & $0.85 \pm 0.62$ & 7.83 & $<0.001$ \\
$\begin{array}{c}\text { Maximum } \\
\left(\times 10^{-3} \mathrm{~mm}^{2} \mathrm{~s}^{-1}\right)\end{array}$ & 1.79 & 2.57 & 2.79 & & \\
$\begin{array}{c}\text { Minimum } \\
\left(\times 10^{-3} \mathrm{~mm}^{2} \mathrm{~s}^{-1}\right)\end{array}$ & 0.78 & 0.29 & 0.40 & & \\
\hline
\end{tabular}

Abbreviation: ADC, apparent diffusion coefficient.

DW images proved accurate in differentiating malignant from benign intratesticular mass lesions in all (100\%) cases in this series.

MR imaging readily identified 41 normal epididymal parts on transverse T2-weighted and DW sequences in this study, as relatively hypointense when compared to the normal testicular parenchyma (Figure 1). The mean \pm s.d. of ADC values $\left(\times 10^{-3} \mathrm{~mm}^{2} \mathrm{~s}^{-1}\right)$ of normal epididymis were $1.39 \pm 0.14$.

Both conventional and DW images proved accurate in characterizing the benign nature of all (100\%) paratesticular lesions in this series. The diagnosis of benignity was confirmed in all patients, by lesion signal hypointensity and hyperintensity on DW sequences and ADC maps, respectively (Figure 4). The mean \pm s.d. of ADC values $\left(\times 10^{-3} \mathrm{~mm}^{2} \mathrm{~s}^{-1}\right)$ of benign paratesticular lesions were $1.72 \pm 0.60$. A difference between the ADC values of the normal epididymis and those of benign paratesticular lesions was noted $(P=0.003)$ (Table 4).

\section{DISCUSSION}

The primary goal in the evaluation of a scrotal mass is defining its location, whether intratesticular or paratesticular and determining the probability of malignancy. The majority of intratesticular masses are malignant and radical orchiectomy is the treatment of choice. However, a confident preoperative characterization of various benign intratesticular entities, including dilatation of rete testis, orchitis, epidermoid cyst, infarction, fibrosis and hematoma, for which radical orchiectomy is unnecessary is important. ${ }^{3-8}$ In these patients, a more conservative approach, as follow-up, tumor enucleation or testissparing surgery may be justified. Sonography, although the primary imaging technique for the evaluation of scrotal pathology, ${ }^{1,2}$ because of its wide availability, low cost and high sensitivity in the identification of scrotal lesions, does not always allow a confident preoperative characterization of scrotal masses. MR imaging of the scrotum has been used as an alternative imaging modality for the evaluation of

Table 4 Independent samples $t$-test showing the difference of mean $A D C$ values between normal epididymis and benign paratesticular lesions

\begin{tabular}{lccc}
\hline & $\begin{array}{c}\text { Normal epididymis } \\
(\mathrm{n}=41)\end{array}$ & $\begin{array}{c}\text { Benign } \\
(\mathrm{n}=8)\end{array}$ & $\mathrm{P}$ \\
\hline $\begin{array}{c}\text { Mean ADC } \pm \text { s.d. } \\
\left(\times 10^{-3} \mathrm{~mm}^{2} \mathrm{~s}^{-1}\right)\end{array}$ & $1.39 \pm 0.14$ & $1.72 \pm 0.60$ & 0.003 \\
$\begin{array}{l}\text { Maximum } \\
\left(\times 10^{-3} \mathrm{~mm}^{2} \mathrm{~s}^{-1}\right)\end{array}$ & 1.69 & 2.66 & \\
$\begin{array}{l}\text { Minimum } \\
\left(\times 10^{-3} \mathrm{~mm}^{2} \mathrm{~s}^{-1}\right)\end{array}$ & 1.19 & 1.08 & \\
\hline
\end{tabular}

Abbreviation: ADC, apparent diffusion coefficient. 
scrotal masses. ${ }^{3-9}$ The technique due to its wide field of view, multiplanar capabilities, high contrast and spatial resolution has proved satisfactory in the localization of a scrotal mass, in the differentiation of paratesticular masses with respect to tumor morphology and tissue characteristics, in the distinction between benign from malignant intratesticular lesions and in the evaluation of the local extent of the disease in cases of testicular carcinomas. ${ }^{3-19}$ The technique is mainly recommended when sonographic findings are inconclusive or inconsistent with clinical findings. ${ }^{5,6}$

In accordance with previous reports, this study demonstrated high accuracy of conventional MR sequences in characterizing both paratesticular and intratesticular mass lesions. ${ }^{4-7}$ The MR criteria used to characterize testicular neoplasms were similar to those presented in the literature, including the presence of a mainly hypointense or a heterogeneous mass lesion on T2-weighted images, inhomogeneously enhancing after contrast material administration. ${ }^{4-7,26,27}$ The coexistence of areas of hemorrhage or necrosis, invasion of testicular tunicae, and extension of the tumor to the paratesticular space and/or the spermatic cord confirmed the diagnosis of malignancy, as it was subsequently proved on histopathology. Although the same group of authors reported the absence of contrast material enhancement as the most sensitive sign in predicting the benign nature of an intratesticular lesion, this study had two false positives, detected as heterogeneously enhancing masses. These cases proved to represent a benign Sertoli cell tumor and postbiopsy changes, respectively.

DW MR imaging watches the random motion of water molecules. In a totally unrestricted background, water movement is completely random, known as Brownian motion or free diffusion. ${ }^{10,11}$ Within biological tissues, it is not completely random, but it is impeded by the interaction with tissue compartments and cell membranes. The extent of tissue cellularity and the presence of intact cell membranes define the impedance of water molecule diffusion. The ADC value relates to the molecular translational movement of water molecules, which is restricted in an environment that contains structures, as cell membranes. It has been reported that high- $b$-value DW imaging depicted high signal intensity for malignant tumors when compared to normal tissues and benign lesions, with lower ADC values for the former, and this was proved also in this material.

In the present study, DW MR imaging proved feasible in differentiating normal scrotal contents from various scrotal diseases. More specifically, all but two testicular malignancies and normal testicular parenchyma were hyperintense on DW images, while all but one benign intratesticular lesions, and all benign paratesticular lesions were hypointense. However, signal intensity of testicular carcinomas was higher compared to normal testis; therefore, visual assessment of the signal intensity on DW images proved feasible in differentiating between normal and cancerous tissue. The reason why normal testis appears hyperintense on DW imaging is probably related to water molecules confined within densely packed seminiferous tubules of normal testicular parenchyma, separated by thin fibrous septa. ${ }^{4}$ In addition, the $\mathrm{ADC}$ values of intratesticular malignancies were lower than that of normal testis and benign lesions and the ADC values of the normal epididymis were lower than that of various benign paratesticular lesions $(P<0.05)$. Therefore, both DW imaging characteristics and ADC calculations of the scrotal contents proved valuable in differentiating between normal and abnormal scrotum, and more important in differentiating normal from cancerous tissue in the testicles. In agreement with previous study, this study reported reduced ADC values for malignancies. ${ }^{12-23}$ The decrease of ADC values in testicular neoplasms is related to the histopathological characteristics of neoplastic tissue, that is, increased tissue cellularity, densely packed malignant cells, enlargement of the nuclei, and angulation of the nuclear contour, all causing reduced motility of water molecules.

DW imaging data should always be interpreted in conjunction with conventional images to achieve a reliable characterization of a lesion, and this was proved also in this study. Based on the intensity characteristics on DW images and calculations of ADC values alone, this series had one false positive in a patient with intratesticular hematoma, related to the low ADC values of bloody products and two false negatives, in patients with multicystic testicular malignancies. However, based on routine MR sequences, the correct diagnosis was possible in these cases. On the contrary, conventional MR data alone characterized incorrectly two heterogeneously enhancing intratesticular masses, subsequently proved benign. The diagnosis of malignancy was not confirmed on DW sequences, due to lesion hypointensity. MR imaging with the interpretation of both conventional and DW data proved accurate in the characterization of all intratesticular and paratesticular lesions in this study.

The use of DW sequences in the evaluation of scrotal pathology may prove valuable, especially in the characterization of lesions that demonstrate equivocal characteristics with routine MR sequences. The addition of DW sequences to routine scanning adds diagnostically useful information with only minimal increase in examination time. The technique can be used in patients with renal insufficiency or a history of allergy to contrast media, because it does not require gadolinium-based contrast agent injection.

In this study, echo planar sequences were used for DW MR maging. The use of these sequences may produce image distortion caused by susceptibility artifacts, due to the presence of air and chemical shift artifacts from fatty tissue. ${ }^{10-23}$ These artifacts were not seen in the present study, probably because of the lack of neighboring organs in the scrotal area. A relatively low signal to noise ratio and spatial resolution has also been reported with these images. However, echo planar imaging with a preparation pulse sensitive to diffusion may yield a satisfactory contrast-to-noise ratio, as proved also in this report. Another advantage of echo planar sequences is rapid image acquisition, reducing motion artifacts. ${ }^{10-23}$

There were some limitations in this study. First, it was a retrospective review of a small number of patients with a narrow range of scrotal abnormalities. Furthermore, although statistically significant differences were noted between the ADC values of normal and abnormal testicular tissue, a wide variation of measurements between individuals was found, so it was difficult to suggest to the ADC cutoff value as an effective parameter for tissue characterization. Prospective studies of large number of patients with various scrotal diseases, including benign intratesticular entities, benign and malignant paratesticular masses are needed to define if MR imaging examination, including DW sequences may improve detection and characterization of scrotal diseases. A possible benign diagnosis based on MR imaging findings may substantially improve patient care, resulting in a reduction of unnecessary radical surgical procedures in these patients. A lesion analysis to compare MR imaging and histopathological findings is also mandatory. Another limitation of this report is that only a consensus reading of the MR imaging data by two radiologists was performed and no interobserver variability was assessed.

In conclusion, DW imaging and ADC values have a potential role in differentiating between normal, benign and malignant scrotal contents, always interpreted in conjunction with the conventional MR imaging sequences. 


\section{AUTHOR CONTRIBUTIONS}

ACT, MIA and KT conceived of the study, participated in its design and helped to draft the manuscript. ACT, MIA, DG, ST and NS participated in data acquisition, analysis and interpretation. ACT and MIA performed the statistical analysis. MIA, NS and KT participated in manuscript revision. All authors read and approved the final manuscript.

\section{COMPETING FINANCIAL INTERESTS}

The authors declare that they have no competing financial interests.

1 Dogra VS, Gottlieb RH, Oka M, Rubens DJ. Sonography of the scrotum. Radiology 2003; 227: 18-36.

2 Hsieh ML, Huang SI, Huang HC, Chen Y, Hsu YC. The reliability of ultrasonographic measurements of testicular volume assessment: comparison of three common formulas with true testicular volume. Asian J Androl 2009; 11: 261-5.

3 Cassidy FH, Ishioka KM, McMahon CJ, Chu P, Sakamoto K et al. MR imaging of scrotal tumors and pseudotumors. Radiographics 2010; 30: 665-83.

4 Woodward PJ, Sohaey R, O'Donoghue MJ, Green DE. Tumors and tumorlike lesions of the testis: radiologic-pathologic correlation. Radiographics 2002; 22: 189-216.

5 Muglia V, Tucci S, Elias J, Trad CS, Bilbey J et al. Magnetic resonance imaging of scrotal diseases: when it makes the difference. Urology 2002; 59: 419-23.

6 Serra AD, Hricak H, Coakley FV, Kim B, Dudley A et al. Inconclusive clinical and ultrasound evaluation of the scrotum: impact on magnetic resonance imaging on patient management and cost. Urology 1998; 51: 1018-21.

7 Tsili AC, Argyropoulou MI, Giannakis D, Sofikitis N, Tsampoulas K. Magnetic resonance imaging in the characterization and local staging of testicular neoplasms. AJR 2010; 194: 682-9.

8 Akbar SA, Sayyed TA, Jafri SZ, Hasteh F, Neil JS. Multimodality imaging of paratesticular neoplasms and their rare mimics. RadioGraphics 2003; 23: 1461-76.

9 Liu HY, Fu YT, Wu CJ, Sun GH. Tuberculous epididymitis: a case report and literature review. Asian J Androl 2005; 7: 329-32.

10 Qayyum A. Diffusion-weighted imaging in the abdomen and pelvis: concepts and applications. RadioGraphics 2009; 29: 1797-810.

11 Saremi F, Knoll AN, Bendavid OJ, Schultze-Haakh H, Narula N et al. Characterization of genitourinary lesions with diffusion-weighted imaging. RadioGraphics 2009; 29: 1295-317.

12 Matsuki M, Inada Y, Tatsugami F, Tanikake M, Narabayashi T et al. Diffusion-weighted MR imaging for urinary bladder carcinoma: initial results. Eur Radiol 2007; 17: 201-4.
13 El-Assmy A, Abou-El-Ghar ME, Mosbah A, El-Nahas AR, Refaie HF et al. Bladder tumour staging: comparison of diffusion and T2-weighted MR imaging. Eur Radiol 2009; 19: 1575-81.

14 Zelhof B, Pickles M, Liney G, Gibbs P, Rodrigues G et al. Correlation of diffusionweighted magnetic resonance data with cellularity in prostate cancer. BJU Int 2009; 103: 883-8.

15 Ren J, Huan $\mathrm{Y}$, Wang $\mathrm{H}, \mathrm{Ge} \mathrm{Y}, \mathrm{Chang} \mathrm{Y}$ et al. Seminal vesicle invasion in prostate cancer: prediction with combined T2-weighted and diffusion-weighted MR imaging. Eur Radiol 2009; 19: 2481-6.

16 Cova M, Squillaci E, Stacul F, Manenti G, Gava S et al. Diffusion-weighted MRI in the evaluation of renal lesions: preliminary results. BRJ Radiol 2004; 77: 851-7.

17 Namimoto T, Awai K, Nakaura T, Yanaga Y, Hirai T et al. Role of diffusion-weighted imaging in the diagnosis of gynecologic diseases. Eur Radiol 2009; 19: 745-60.

18 Lim HK, Kim JK, Kim KA, Cho KS. Prostate cancer: apparent diffusion coefficient map with T2-weighted images for detection—a multireader study. Radiology 2009; 250: 145-51.

19 Whittaker CS, Coady A, Culver L, Rustin G, Padwick M et al. Diffusion-weighted MR imaging of female pelvic tumors: a pictorial review. RadioGraphics 2009; 29: 759_ 78.

20 Inada Y, Matsuki M, Nakai G, Tatsugami F, Tanikake M et al. Body diffusion-weighted MR imaging of uterine endometrial cancer: is it helpful in the detection of cancer in nonenhanced MR imaging? Eur J Radiol 2009; 70: 122-7.

21 Chen J, Zhang V, Liang B, Yang Z. The utility of diffusion-weighted MR imaging in cervical cancer. Eur J Radiol 2010; 74: 101-6.

22 Fujii S, Matsusue E, Kigawa J, Sato S, Kanasaki V et al. Diagnostic accuracy of the apparent diffusion coefficient in differentiating benign from malignant uterine endometrial cavity lesions: initial results. Eur Radiol 2008; 18: 384-9.

23 Fujii S, Matsusue E, Kanasaki Y, Kanamori V, Nakanishi J et al. Detection of peritoneal dissemination in gynaecological malignancy: evaluation by diffusion-weighted MR imaging. Eur Radiol 2008; 18: 18-23.

24 Kangasniemi M, Kaipia A, Joensuu R. Diffusion weighted magnetic resonance imaging of rat testes: a method for early detection of ischemia. J Urol 2001; 166: 2542-4.

25 Toshiki K, Yoshiyuki K, Yasuhiro S, Makoto I, Kentaro M et al. Usefulness of MR fatsuppressed T2-weighted and diffusion-weighted imaging for the diagnosis of nonpalpable testes. J Urol 2008; 179: 387-8.

26 Johnson JO, Mattrey RF, Phillipson J. Differentiation of seminomatous from nonseminomatous testicular tumors with MR imaging. AJR 1990; 154: 539-43.

27 Tsili AC, Tsampoulas C, Giannakopoulos X, Stefanou D, Alamanos Y et al. MRI in the histologic characterization of testicular neoplasms. AJR 2007; 189: W331-7.

28 Rouviere O, Bouvier R, Pangaud C, Jeune M, Dawahra M et al. Tubular ectasia of the rete testis: a potential pitfall in scrotal imaging. Eur Radiol 1999; 9: 1862-8.

29 Tartar VM, Trambert MA, Balsara ZN, Mattrey RF.Tubular ectasia of the testicle: Sonographic and MR imaging appearance. AJR 1993; 160: 539-42. 